\section{Quantification of the foreign body reaction by means of a miniaturized imaging window for intravital nonlinear microscopy}

Claudio Conci, 1 Emanuela Jacchetti, 1

Tommaso Zandrini, ${ }^{1}$ Laura Sironi, ${ }^{2}$ Maddalena Collini, 2 Giuseppe Chirico, ${ }^{2}$ Giulio Cerullo, ${ }^{3}$ Roberto Osellame, ${ }^{3}$ Manuela Teresa Raimondi ${ }^{1}$

1Department of Chemistry, Materials and Chemical Engineering Giulio Natta, Politecnico di Milano, Milan; 2Department of Physics, University of Milano-Bicocca, Milan; ${ }^{3}$ Istituto di Fotonica e Nanotecnologie (IFN)-CNR and Department of Physics, Politecnico di Milano, Milan, Italy

\begin{abstract}
Brand new biomaterials, intended to be used on humans, must undergo in vivo quantification standardized, expensive and unethical procedures mainly based on histopathological analysis, from dissections, as defined by the ISO 10993 normative set. The aim is to prove the biomaterials biocompatibility. There exist no methods based on intravital microscopy able to satisfy the normative quantification requirements both reducing the number of employed animals and related costs. We developed a miniaturized imaging window, the Microatlas, which allows subcutaneous repeated observations in vivo of the foreign body reactions, for example to the implantation of a biomaterial. Confocal and twophoton microscopy inspections at Microatlas implantation sites demonstrated growth of the recipient tissue inside the microgrids both with micro vascularization formation and collagen generation. In conclusion, the Microatlas guided in vivo a quantifiable localized reaction inside its microscaffold, both in terms of cell repopulation, collagen and capillary formation as a probable foreign body reaction.
\end{abstract}

\section{Introduction}

To gain the authorization of being used on humans, brand new biomaterials must undergo in vivo quantification standardized, expensive and unethical procedures (defined by the ISO 10993) to prove their biocompatibility. These ones are mainly based on histopathological analyses in large number of mammalians, subcutaneously implanted, through different timepoints. Currently there exist no methods based on intravital microscopy able to satisfy the normative quantification requirements, with the aim to reduce the number of employed animals and related costs. We developed a miniaturized imaging window, the Microatlas, which can be implanted subcutaneously and allows repeated observation in vivo of the foreign body reactions, for example to the implantation of a biomaterial. The device hosts a miniaturized scaffold able to guide cell migration close to the desired target. By applying two-photon fluorescence microscopy to the Microatlas, once implanted in vivo and repopulated by cells and blood vessels, it is possible to observe and quantify the foreign body reactions in the same animal and tissue district, at different time points. Thus, we can reduce the number of employed animals in subcutaneous validation protocols, refine and boost the conducted validation analyses and replace old and outdated quantification processes in term of cellular density, blood vessels sprouting, collagen and fatty infiltrate generation. Here, we grafted the Microatlas in living chicken embryos to conduct in vivo validation assays.

\section{Materials and Methods}

The Microatlas micro scaffolds were fabricated by two-photon laser polymerization on circular glass coverslips (Ø:5-12 $\mathrm{mm})$, with a biocompatible photoresist, SZ2080. The micro scaffolds consist in several micro grids $(500 \mu \mathrm{m} \times 500 \mu \mathrm{m} \times 100$ $\mu \mathrm{m})$. Reference structures were integrated to allow the microscope field-of-view repositioning at different time-points (Figure 1). The chicken embryo ex ovo culture was optimized and the optimal implantation
Correspondence: Claudio Conci, Department of Chemistry, Materials and Chemical Engineering Giulio Natta, Politecnico di Milano, Milan, Italy.

E-mail: claudio.conci@polimi.it

Key words: Imaging window; nonlinear microscopy; two-photon polymerization.

Acknowledgments: this project is funded by the Italian Ministry of University and Research (call MIUR-FARE 2016, project BEYOND, Cod. R16ZNN2R9K).

Conference presentation: this paper was presented at the Second Centro 3R Annual Meeting - 3Rs in Italian Universities, 2019 , June 20-21, University of Genoa, Italy.

Received for publication: 28 October 2019

Accepted for publication: 11 November 2019

This work is licensed under a Creative Commons Attribution NonCommercial 4.0 License (CC BY-NC 4.0).

CC Copyright: the Author(s), 2019

Licensee PAGEPress, Italy

Biomedical Science and Engineering 2019; 3(s3):106 doi:10.4081/bse.2019.106

time points were selected. The Microatlas was implanted and it was inspected by twophoton fluorescence and confocal microscopy. At each time point, the embryo was two-photon imaged first, then formalinfixed, labelled with DRAQ5TM and imaged in confocal microscopy.

\section{Results}

Confocal and two-photon inspections at Microatlas implantation sites demonstrated growth of the recipient tissue inside the micro grids both with micro vascularization formation. Two-photon fluorescence acqui 
A

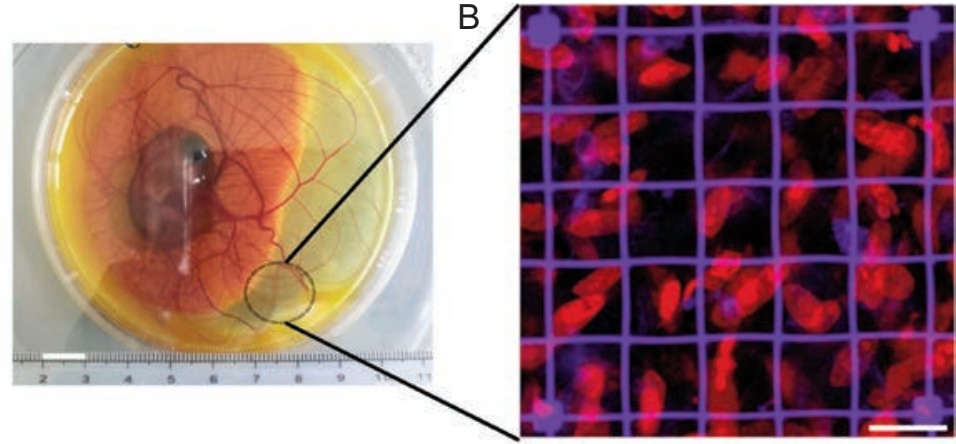

C

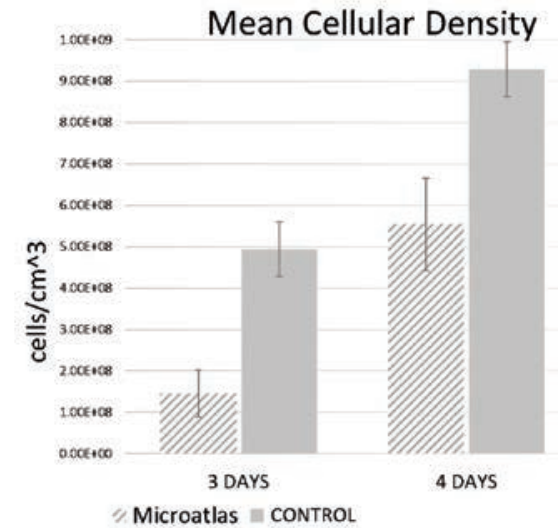

Figure 2. A) Chick embryo at incubation day 11 , scalebar $1 \mathrm{~cm}$. B) cells imaged inside the Microatlas, scalebar $20 \mu \mathrm{m}$. C) cellular density trend inside the Microatlas microgrids. sitions of label-free specimens specifically showed the presence of a layer of collagen type I, localized mainly around and inside the implanted Microatlas. Microscope images allowed quantification of cell density, collagen formation and neo-vascularization rate (Figure 2) inside the Microatlas as required by the ISO10993-6.

\section{Conclusions}

The Microatlas guided in vivo a quantifiable localized reaction inside its microscaffold, both in terms of cell repopulation, collagen generation and capillary formation as a probable foreign body reaction. Thus, our device can be used as a powerful imaging window for intravital fluorescence microscopy with the capability to quantify in vivo the reaction to biomaterial implantation. 